\title{
Beliefs about Emotions, Depression, Anxiety and Fatigue: A Mediational Analysis
}

\author{
Mia Sydenham, Jennifer Beardwood and Katharine A. Rimes \\ Institute of Psychiatry, Psychology and Neuroscience (IoPPN), King's College London, UK
}

\begin{abstract}
Background: Beliefs that it is unacceptable to experience or express negative emotions have been found to be associated with various clinical problems. It is unclear how such beliefs, which could be viewed as a form of unhelpful perfectionism about emotions, may contribute to symptomatology. Aims: This study investigated two hypotheses: a) greater endorsement of beliefs about the unacceptability of negative emotions will be associated with greater emotional avoidance and lower levels of support-seeking and self-compassion; $b$ ) these beliefs about emotions will be associated with higher levels of symptoms of depression, anxiety and fatigue and that this relationship will be mediated by social support-seeking, emotional avoidance and self-compassion. Method: Online questionnaires were completed by 451 community participants. Mediational analyses were undertaken to investigate emotional avoidance, social support-seeking and self-compassion as mediators of the relationship between beliefs about emotions and symptoms of depression, anxiety and fatigue. Results: Beliefs about the unacceptability of negative emotions were significantly associated with more emotional avoidance and less self-compassion and support-seeking. The relationships between beliefs about emotions and depression, anxiety and fatigue were significantly mediated by selfcompassion and emotional avoidance but not social support-seeking. Conclusions: Future research should investigate whether interventions that pay particular attention to emotional avoidance and self-compassion, such as mindfulness-based therapy or modified forms of CBT, may be beneficial in reducing distress and fatigue associated with beliefs about the unacceptability of negative emotions.
\end{abstract}

Keywords: Emotional avoidance, emotional processing, help-seeking, perfectionism, selfcompassion, social support.

\section{Introduction}

Beliefs that it is unacceptable to experience or express negative emotions, which could be viewed as a form of maladaptive perfectionism about emotions, have been reported to be elevated in a range of clinical problems, including eating disorders, anxiety and depression (Campbell-Sills, Barlow, Brown and Hoffman, 2006) and chronic fatigue syndrome (Rimes and Chalder, 2010). Although it is possible that the experience of such problems may result in these beliefs becoming more negative, it is also possible that they contribute to the development or maintenance of such problems. For example, individuals with these

Correspondence to Katharine Rimes, Institute of Psychiatry, Psychology and Neuroscience, Department of Psychology, De Crespigny Park, London SE5 8AF, UK. E-mail: katharine.rimes@kcl.ac.uk 
beliefs about emotions may avoid social support-seeking to prevent others from knowing that they are experiencing negative emotions. However, this means that the individual fails to receive support that could alleviate their distress. Individuals may try to further hide their negative feelings by avoiding their emotions (Hambrook et al., 2011). Unfortunately, emotional avoidance strategies such as emotional suppression can lead to an increase in associated sympathetic arousal, which can increase the intensity of experiencing a negative emotion, in turn causing more distress for the individual (Campbell-Sills et al., 2006). Another possible mediator in the relationship between perfectionist beliefs about emotions and clinical problems is self-compassion. Neff (2003) suggests that self-compassionate individuals are more accepting when they fail to meet their personal goals, and thus experience less distress. This is supported by evidence that general maladaptive perfectionism is negatively associated with self-compassion, which in turn is associated with a range of clinical problems, including depression and anxiety (Neff, 2003). No previous research has examined whether the relationship between beliefs about the unacceptability of negative emotions and clinical symptoms are mediated by support-seeking, emotional avoidance or self-compassion. The present study investigates the hypothesis that individuals with stronger beliefs about the unacceptability of expressing or experiencing negative emotions will seek less social support, report greater emotional avoidance and lower levels of self-compassion. Second, it is predicted that these beliefs about emotions will be associated with higher levels of symptoms of depression, anxiety and fatigue and that this relationship will be mediated by social supportseeking, emotional avoidance and self-compassion.

\begin{abstract}
Method
Design

A cross-sectional, questionnaire-based design via an online survey was used.
\end{abstract}

\title{
Participants
}

The sample of 451 adults consisted of 330 females and 116 males ( 5 respondents did not provide their gender) with an age range of 17 to 69 years $(M=27.3, S D=11.0 ; 11$ respondents did not provide their age). Participants were only included if they had completed all of the questionnaires.

\section{Measures}

Participants completed the following questionnaires: 1) Beliefs about Emotions Scale (BES) (Rimes and Chalder, 2010, current $\alpha=.91$ ): assessing beliefs about the unacceptability of experiencing and expressing negative emotions; 2) Berlin Support Seeking Scale (BSSS) (Schwarzer and Schulz, 2000, current $\alpha=.82$ ): a 5-item subscale from the BSSS that assesses cognitive and behavioural aspects of social support-seeking. 3) Emotional Avoidance Questionnaire (EAQ) (Taylor et al., 2004, current $\alpha=.90$ ): assessing emotional avoidance, with three items removed as they mention beliefs and hence potentially overlap with the BES; 4) Self-Compassion Scale (short form) (SCS) (Raes, Pommier, Neff and Van Gucht, 2011, current $\alpha=.85$ ): measuring self-compassion; 5) Patient Health Questionnaire (PHQ-9) 
(Kroenke, Spitzer and Williams, 2001, current $\alpha=.90$ ): measuring depressive symptoms over the past 2 weeks; 6) Generalized Anxiety Disorder Questionnaire (GAD7) (Spitzer, Kroenke, Williams and Lowe, 2006, current $\alpha=.90$ ): measuring symptoms of anxiety over the past 2weeks; 7) The physical fatigue subscale of the Chalder Fatigue Scale (CFS) (Chalder et al., 1993, current $\alpha=.91$ ): measuring physical fatigue. For each measure, high scores indicate higher levels of each construct.

\section{Procedure}

Ethical approval was gained from King's College London Research Ethics Committees (CREC) (PNM/13/14-50). Participants were recruited via opportunity sampling through university e-mails, online research recruitment sites and social networking sites. The study was conducted online via the anonymous survey.

\section{Data preparation and statistical analysis}

Missing items within a scale were replaced via mean substitution. If more than $25 \%$ of data were missing for any scale, the respondent's data were not included in this study. Data were analysed using SPSS Version 22. Mediation analysis was undertaken using a bootstrapping method with the SPSS macro "PROCESS" (Hayes, 2014; http://www.processmacro.org/ download.html). The variables self-compassion, emotional avoidance and social supportseeking were entered simultaneously into the mediation model. Scores on the Beliefs about Emotions Scale were the independent variable. Mediation analysis was carried out separately for each of the outcome variables (depression, anxiety, and fatigue); 95\% confidence intervals were computed and results are based on 5000 bootstrapped samples.

\section{Results}

\section{Correlational findings}

Pearson correlations indicated that unhelpful beliefs about emotions were significantly associated with greater emotional avoidance $(r=.51, p<.001)$, lower social support-seeking $(r=-.41, p<.001)$ and lower self-compassion $(r=-.45, p<.001)$, as well as with greater symptoms of depression $(r=.33, p<.001)$, anxiety $(r=.32, p<.001)$ and fatigue $(r=.16$, $p<.001)$.

\section{Mediational findings}

For each mediation model, beliefs about emotions were significantly associated with selfcompassion, emotional avoidance and social support-seeking. The total effect of beliefs about emotions on each of the outcome variables was significant and the direct effect nonsignificant. Emotional avoidance and self-compassion significantly predicted depression, anxiety and fatigue. The confidence intervals for the indirect effects of both of these mediating variables on the three outcome variables excluded zero, thus both emotional avoidance and self-compassion significantly mediated the relationship between beliefs about emotions and depression, anxiety and fatigue. Social support-seeking did not significantly predict 


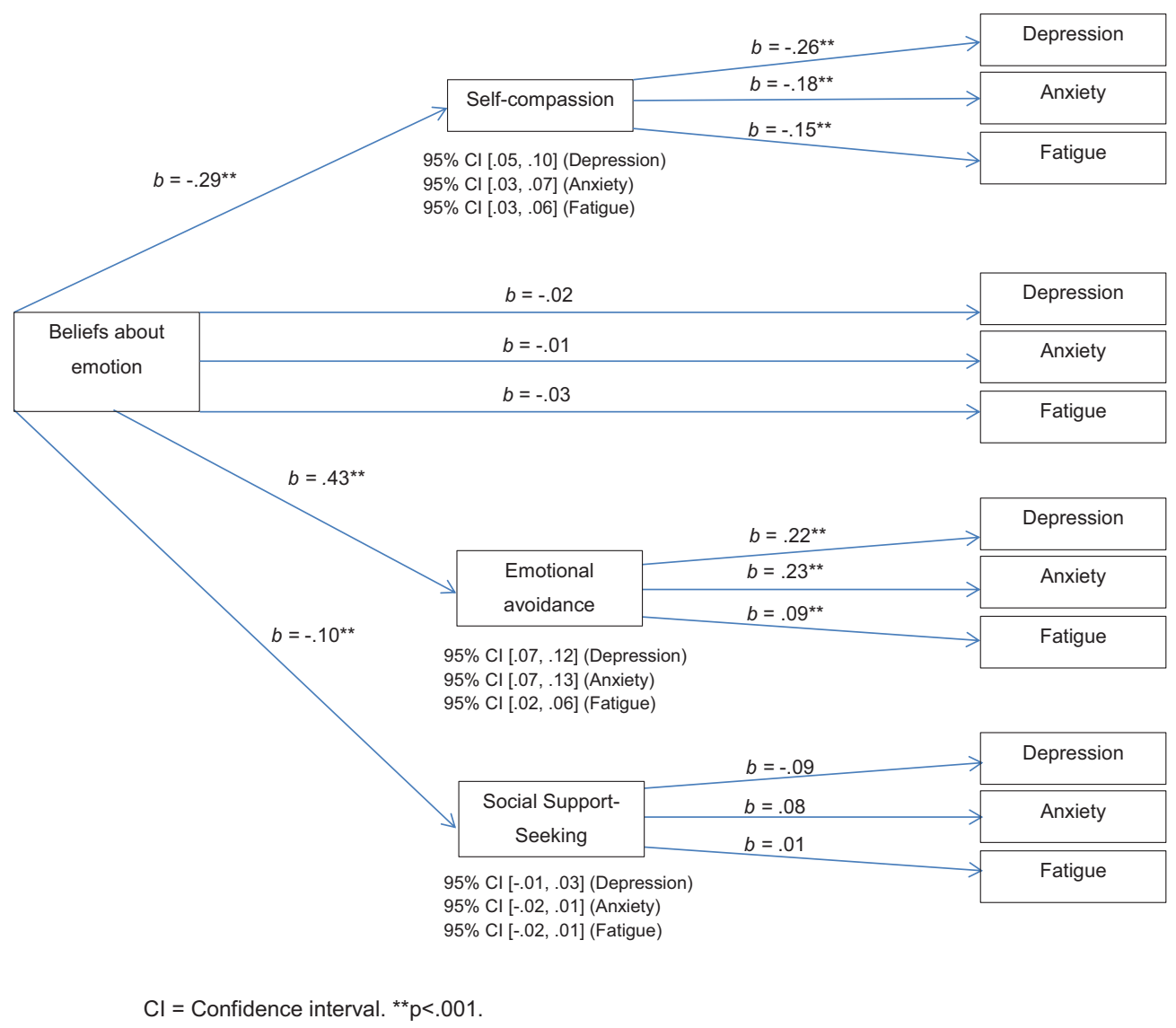

Figure 1. (Colour online) Mediation models for depression, anxiety and fatigue $(\mathrm{n}=451)$.

depression, anxiety or fatigue and included zero within its confidence intervals, thus did not significantly mediate the relationship between beliefs about emotions and depression, anxiety or fatigue. See Figure 1 for the mediation models for depression, anxiety and fatigue.

\section{Discussion}

As predicted, stronger beliefs about the unacceptability of experiencing and expressing negative emotions were associated with more emotional avoidance and less self-compassion and support-seeking. The relationships between beliefs about emotions and depression, anxiety and fatigue were significantly mediated by self-compassion and emotion avoidance. However, contrary to expectations, social support-seeking was not a significant mediator in this relationship. The finding that unhelpful beliefs about emotions were positively associated with emotional avoidance supports previous research (Campbell-Sills et al., 2006). The current finding that emotional avoidance mediates the relationship between unhelpful beliefs about emotions and depression, anxiety and fatigue suggests that encouraging the acceptance 
of negative emotions may be helpful in reducing distress or fatigued in individuals with such beliefs about their emotions. Self-compassion was also a mediator of the relationship between beliefs about the unacceptability of negative emotions and depression, anxiety and fatigue, in line with the finding that self-compassion mediates the relationship between general maladaptive perfectionist beliefs and anxiety and depression (e.g. Neff, 2003). Unhelpful beliefs about emotions were associated with lower levels of social support-seeking; however social support-seeking did not mediate the relationship between perfectionist beliefs about emotions and depression, anxiety or fatigue, suggesting that reduced social support-seeking is not an important mechanism in this relationship.

CBT for chronic fatigue syndrome has been associated with a reduction in unhelpful beliefs about emotions (Rimes and Chalder, 2010), as has mindfulness-based cognitive behaviour therapy (MBCT; Rimes and Wingrove, 2013). As MBCT has a particular focus upon self-compassion and the acceptance of emotions (Rimes and Wingrove, 2013), it may be particularly beneficial in reducing the clinical outcomes associated with perfectionist beliefs about emotions. Future research could investigate whether MBCT for depression or anxiety reduces beliefs about the unacceptability of negative emotions and whether this change mediates clinical outcomes.

\section{Limitations}

The study had a number of limitations including a female gender bias that may limit generalizability of the findings. The use of an online methodology with community participants enabled a large sample size, but it cannot be assumed that the results would generalize to a clinical population. Furthermore, as a cross-sectional design was used, causality between the variables cannot be established, thus mediation reflected statistical mediation only.

\section{Conclusions}

The present study suggests that the relationship between beliefs about the unacceptability of negative emotions and depression, anxiety and fatigue is mediated by lower levels of self-compassion and greater levels of emotional avoidance. Social support-seeking was not found to be a significant mediator of this relationship. Therapies suited to addressing perfectionist beliefs about emotions for those experiencing clinical issues include MBCT, which encourages openness to emotions and self-compassion specifically as core elements of the treatment.

\section{Acknowledgements}

Conflict of interest: The authors have no conflicts of interest with respect to this publication.

\section{Supplementary material}

An extended version is also available online under the Brief Clinical Report Supplementary Materials tab in the table of contents. Please visit http://dx.doi.org/10.1017/ S1352465816000199 


\section{References}

Campbell-Sills, L., Barlow, D. H., Brown, T. A. and Hofmann, S. G. (2006). Acceptability and suppression of negative emotion in anxiety and mood disorders. Emotion, 6, 587-595.

Chalder, T., Berelowitz, G., Pawlikowska, T., Watts, L., Wessely, S., Wright, D., et al. (1993). Development of a fatigue scale. Journal of Psychosomatic Research, 37, 147-153.

Hambrook, D., Oldershaw, A., Rimes, K., Schmidt, U., Tchanturia, K., Treasure, J., et al. (2011). Emotional expression, self-silencing, and distress tolerance in anorexia nervosa and chronic fatigue syndrome. British Journal of Clinical Psychology, 50, 310-325.

Kroenke, K., Spitzer, R. L. and Williams, J. B. (2001). The PHQ-9. Journal of General Internal Medicine, 16, 606-613.

Neff, K. D. (2003). The development and validation of a scale to measure self-compassion. Self and Identity, 2, 223-250.

Raes, F., Pommier, E., Neff, K. D. and Van Gucht, D. (2011). Construction and factorial validation of a short form of the self-compassion scale. Clinical Psychology and Psychotherapy, 18, 250-255.

Rimes, K. A. and Chalder, T. (2010). The Beliefs about Emotions Scale: validity, reliability and sensitivity to change. Journal of Psychosomatic Research, 68, 285-292.

Rimes, K. A. and Wingrove, J. (2013). Mindfulness-based cognitive therapy for people with chronic fatigue syndrome still experiencing excessive fatigue after cognitive behaviour therapy: a pilot randomized study. Clinical Psychology and Psychotherapy, 20, 107-117.

Schwarzer, R. and Schulz, U. (2000). Berlin Social Support Scales (BSSS). Available at: www.coping.de

Spitzer, R. L., Kroenke, K., Williams, J. B. and Löwe, B. (2006). A brief measure for assessing generalized anxiety disorder: the GAD-7. Archives of Internal Medicine, 166, 1092-1097.

Taylor, C. T., Laposa, J. M. and Alden, L. E. (2004). Is avoidant personality disorder more than just social avoidance? Journal of Personality Disorders, 18, 571-594. 